\title{
Throughput Analysis for Cognitive Radio Networks with Multiple Primary Users and Imperfect Spectrum Sensing
}

\author{
Wuchen Tang*, Muhammad Zeeshan Shakir ${ }^{\dagger}$, Muhammad Ali Imran*, \\ Rahim Tafazolli*, and Mohamed-Slim Alouini ${ }^{\dagger}$ \\ ${ }^{*}$ Centre for Communication Systems Research (CCSR), University of Surrey \\ Guildford GU2 7XH, Surrey, United Kingdom \\ Email:\{w.tang, m.imran, r.tafazolli\}@ surrey.ac.uk \\ $\dagger^{\dagger}$ Division of Physical Sciences and Engineering, KAUST \\ Makkah Province, Thuwal 23955-6900, Kingdom of Saudi Arabia \\ Email: \{muhammad.shakir, slim.alouini\}@kaust.edu.sa
}

\begin{abstract}
In cognitive radio networks, the licensed frequency bands of the primary users (PUs) are available to the secondary user (SU) provided that they do not cause significant interference to the PUs. In this paper, we analyzed the normalized throughput of the SU with multiple PUs coexisting under any Frequency Division Multiple Access (FDMA) communication protocol. We consider a cognitive radio transmission where the frame structure consists of sensing and data transmission slots. In order to achieve the maximum normalized throughput of the SU and control the interference level to the legal PUs, the optimal frame length of the SU is found via simulation. In this context, a new analytical formula has been expressed for the achievable normalized throughput of SU with multiple PUs under prefect and imperfect spectrum sensing scenarios. Moreover, the impact of imperfect sensing, variable frame length of SU and the variable PU traffic loads, on the normalized throughput has been critically investigated. It has been shown that the analytical and simulation results are in perfect agreement. Our analytical results are much useful to determine how to select the frame duration length subject to the parameters of cognitive radio network such as network traffic load, achievable sensing accuracy and number of coexisting PUs.
\end{abstract}

Index Terms

Cognitive radio; normalized throughput; imperfect spectrum sensing; probability of collision; traffic 
load.

\section{INTRODUCTION}

Spectrum bands are considered as one of the important limited resources in wireless communications which should be utilized efficiently. However, it is reported by Federal Communications Commission (FCC) that $70 \%$ of the allocated spectrum bands in US are not fully utilized [1]. Under this motivation, thanks to cognitive radio technology, the secondary user (SU) is allowed to use the free spectrum bands which are licensed originally to the primary users (PUs) [2-5]. In order to avoid the intolerable interference to the PUs, it is required that the spectrum sensing should be carried out more frequent to control the SU data transmission and to achieve the maximum throughput.

In cognitive radio networks, the throughput of the $\mathrm{SU}^{1}$ is an important parameter which should be maximized under the condition that the interference level to the PU should be below a certain threshold. In this context, a typical frame structure is considered for the SU which comprises of the sensing and the data transmission slots. The sensing slot and the data transmission slot are required to be coordinated in a unit frame such that (i) the amount of transmitted data packets increases and (ii) the number of collisions with the PUs decreases.

The length of the sensing slot determines the accuracy of spectrum sensing detection such that the higher accuracy can be obtained by collecting sufficient number of samples during the sensing phase, i.e. increasing the sensing length [6-8]. To provide higher sensing accuracy, several spectrum sensing algorithms have been introduced and developed, such as the traditional energy detector and eigenvalue-based detection algorithms which are based on the eigenvalues of the received signal covariance matrix [6-11]. From the SUs' perspective, the lower probability of sensing errors mandates to improve the throughput of the SU. Therefore, a tradeoff is required to be defined between the sensing length and throughput of the SUs based on the frame structure $[12,13]$. Following each sensing period, the secondary transmission starts when

\footnotetext{
${ }^{1}$ In cognitive radio network, the throughput gain of the network mainly depends on the throughput gain of SUs because the throughput of PUs are not expected to be affected by the SUs activities. Hence, in this work we consider the throughput of cognitive radio network as the throughput of SUs only.
} 
the licensed channel is considered as idle by the SU. Otherwise, the SU has to wait until the next frame to sense the licensed channels again before any opportunistic usage of channels. In [13], the optimization of spectrum sensing length has been studied using the sensing-throughput tradeoff metric. Specifically, the paper studied the design of the sensing length to maximize the achievable throughput of a single channel cognitive radio network, under the constraint of the probability of detection. To provide better service for SUs, it is advisable to aggregate the perceived spectrum opportunities obtained through simultaneous sensing over multiple channels. In [14], the design of the sensing time has been investigated in order to maximize the average achievable throughput of the multiple channels in cognitive radio network without causing harmful interference to the PUs or exceeding the power limit of the secondary transmitter. The optimal sensing length is identified for the above problem under average power constraint. As an extended work of [14], authors in [15] also studied the problem of designing the optimal sensing length that maximizes the throughput of a wideband sensing-based spectrum sharing cognitive radio network and a wideband opportunistic spectrum access cognitive radio network. Different from [14], in [15] the authors introduce an average interference power constraint in the wideband opportunistic spectrum access scheme (besides the average transmit power constraint), in order to effectively protect the PUs from harmful interference for the realistic scenario of imperfect sensing. Moreover, the effect of this constraint on the optimal sensing time has also been demonstrated in paper.

Compared with sensing length, transmission duration length also impacts the extent of interference between the PUs and the SU and mainly dominates the throughput of the SU. Therefore, the optimization of transmission duration length should also be investigated in order to further improve throughput for higher quality networks. However, current work on the impact of data transmission length on the throughput is limited. With the same frame structure, [16] considered a cognitive radio network that a SU makes opportunistic access to a spectrum band which is legally licensed to a PU according to the sensing result. Based on the required sensing time and the traffic pattern of PU, an optimal value for transmission duration of SU has been determined such that the throughput of the SU is maximized. However, in [16], the data transmission length is 
optimized only with single PU coexisting in the cognitive radio network. Moreover, the analysis is based on the assumption that the spectrum sensing by the SU is perfect.

In this paper, we consider a variable data transmission length with constant sensing length which also means variable frame length. Moreover, we analyze the normalized throughput of the SU who is allowed to make opportunistic access to the spectrum bands which are originally licensed to the multiple PUs coexisting in the network with a frequency division multiplexing system, which means the PUs and channels are one to one mapping. The contributions of this work are described as:

- we extend the analytical analysis for both perfect and imperfect spectrum sensing by the SU and provide the analytical expression of normalized throughput for the SU with multiple PUs coexisting in the cognitive networks.

- we address the selection of optimal frame length of the SU cognitive transmission under variable traffic load of PUs.

- we investigate the impact of imperfect sensing, variable frame length of SU and the variable PU traffic loads, on the normalized throughput.

- we study the selection of the frame duration length and required sensing accuracy subject to the parameters of cognitive radio network such as network traffic load and number of coexisting PUs.

The rest of this paper is organized as follows. Section II defines the system model and explains how the SU detects and accesses the multiple licensed spectrum bands. In Section III, we provide the normalized throughput expression of the SU for perfect and imperfect spectrum sensing cases with multiple PUs. Simulation results and discussions to validate the analytical analysis are presented in Section IV. Finally, we conclude the paper in Section V.

\section{SYSTEM MODEL}

In this paper, we consider a single SU coexisting with $K$ PUs in the cognitive radio network, which means there are $K$ channels available for the SU to sense and to access, if available. The SU may be considered as a secondary terminal with $M$ transmit antennas such that the SU collects 
$N$ samples from each PU during the sensing phase for each sensing. The collected samples will be forwarded to a fusion center for combined processing and decision. A typical frame structure of the SU is shown in Fig. 1 where each frame with length $T$ consists of the sensing slot with length $\tau$ and the data transmission slot with length $T-\tau$. The data transmission of the SU is activated subject to the spectrum sensing results based on the following two hypotheses for each channel

$$
\begin{aligned}
& \mathcal{H}_{0}: \boldsymbol{y}(n)=\boldsymbol{w}(n), \\
& \mathcal{H}_{1}: \boldsymbol{y}(n)=\boldsymbol{h}(n) s(n)+\boldsymbol{w}(n),
\end{aligned}
$$

where $\boldsymbol{y}(n)=\left[y_{1}(n), \cdots, y_{M}(n)\right]^{T}$ is the $M \times 1$ observed complex time series containing $M$ samples received at instant $n ; \boldsymbol{w}(n)$ for all $n=\{1,2, \cdots, N\}$ represents a $M \times 1$ complex circular Gaussian white noise process with unknown variance $\sigma_{w}^{2}$. In (2), the vector $\boldsymbol{h}(n) \in \mathbb{C}^{M \times 1}$ typically represents the propagation channel between the corresponding PU and $M$ antennas and the signal $s(n)$ for all $n=\{1,2, \cdots, N\}$ denotes a standard scalar i.i.d circular complex Gaussian process with zero mean and unit variance and stands for the source signal to be detected. Hypothesis $\mathcal{H}_{0}$ and $\mathcal{H}_{1}$ stand for the spectrum band detected are idle and occupied respectively.

The SU transmits data during the data transmission phase only when the sensing result during the sensing phase is $\mathcal{H}_{0}$, otherwise the SU will keep silent until the next frame. A typical cognitive radio transmission with two PUs of such system model is shown in Fig. 2. If more than one spectrum bands of PUs are sensed idle (available) to the SU, it is assumed that the SU chooses the best channel to transmit data packets. The quality of channel could be estimated by very short time compared with the sensing length. For example, in IEEE 802.11a, only 4 pilot symbols are used for channel estimation [17] while in IEEE 802.22 wireless regional area network (WRAN) thousands of samples are required for a typical sensing [18]. Therefore, for the frame structure of SU, the channel estimation time could be ignored and thus is not considered in Fig. 1.

Furthermore, we assumed (i) the traffic loads of the PUs are exponentially distributed with 
the mean of the occupied and the idle durations denoted by $\alpha_{1}$ and $\alpha_{0}$ respectively, (ii) the SU is heavily loaded and always has packets to transmit. The packet collisions during the transmission are considered as interference since the collided packets are assumed to be lost. In this context, in order to maximize the throughput, the interference should be minimized. For the perfect spectrum sensing case, collisions occur only when the corresponding PU activate during the data transmission phase of the SU, which is illustrated by Fig. 3. While for the imperfect spectrum sensing scenario, there are extra collisions which occur due to false detection of the spectrum, i.e. the situation where the spectrum is actually occupied by the PUs and wrongly detected as idle which is referred to as missed detection. It is to note that multiple access control can also avoid data collision, but mainly when multiple SUs are considered. If more than one SUs try to access the same channel, multiple access control may select the proper SU to avoid the collisions between SUs. The proper SU selection may also further reduce the collisions between the selected SU and PU. Some similar works have been done on this issue such as $[19,20]$. However, in this paper we assume only one SU existing in the cognitive radio network and therefore the collisions are between the SU and the PUs. Moreover, another type of spectrum sensing errors which is referred to as false alarm further decreases the throughput of the cognitive radio transmission of the SU and reduces the spectrum utilization efficiency. Therefore, it is important to discover the degradation to the throughput due to imperfect sensing under variable traffic loads of PUs.

\section{NORMALIZED ThroughPUT FOR THE SU}

We consider there are $K$ PUs and each of the $K$ channels has the same channel capacity which is denoted as $C$. Let us define $P_{p}^{s}$ and $P_{i p}^{s}$ as the probability of the collision for the SU during the cognitive radio transmission when no sensing error occurs and when missed detection occurs respectively. Analytical expressions of the throughput for the SU under these two scenarios will be given in the following sub-sections. 


\section{A. Throughput of SU with Perfect Spectrum Sensing}

With perfect spectrum sensing, the sensing results are always accurate. The SU will keep silent only if all channels are occupied, otherwise the SU will transmit data during the data transmission phase. If we assume that $i$ indicates the number of occupied channels while $K-i$ indicates the number of free (idle) channels, where $i=0, \ldots, K$. For any sensing instant, the probability that there are $i$ occupied channels and $K-i$ free channels is determined by the traffic density of the PUs and could be expressed as

$$
P_{(K, i)}=(1-\beta)^{K-i} \beta^{i},
$$

where $\beta=\left(\frac{\alpha_{1}}{\alpha_{0}+\alpha_{1}}\right)$ is the traffic load factor which is defined as the amount of the traffic that each PU generates on the respective channel [21]; as mentioned in last section, $\alpha_{1}$ and $\alpha_{0}$ are the mean of the occupied and the idle durations of PUs traffic loads, respectively. Similarly, for any sensing instant, the probability that the SU senses at least one free channel and is allowed to access one of them, is given by

$$
P_{(K, i<K)}=\left(1-\beta^{K}\right) .
$$

Now the throughput of the SU can be expressed as

$$
R_{p}^{*}(K, T)=P_{(K, i<K)} \frac{T-\tau}{T}\left(1-P_{p}^{s}\right) C,
$$

and the normalized throughput may be expressed as

$$
R_{p}(K, T)=P_{(K, i<K)} \frac{T-\tau}{T}\left(1-P_{p}^{s}\right),
$$

where $P_{(K, i<K)}$ is given as (4). It is to note that since the length of the sensing slot is fixed, the longer the frame duration $T$, the higher the probability that the PU will become active during data transmission phase, which leads to more collisions for both the PU and the SU thus decreasing the throughput of the SU. To this extent, we may now calculate the $P_{p}^{s}$ to obtain the normalized throughput expression of the cognitive radio transmission of SU under perfect spectrum sensing 
with multiple PUs.

We define $t$ as the time required for PU to become active from starting point of data transmission slot in the current frame, which is shown in Fig. 2. The collision duration of the current frame with PU can be expressed as

$$
y(t)= \begin{cases}T-\tau-t, & 0 \leq t \leq(T-\tau) \\ 0, & t \geq(T-\tau)\end{cases}
$$

Because the random variable $t$ is exponential distributed, the probability density function (PDF) of $t$ is

$$
P(t)=\frac{1}{\alpha_{0}} \exp \left(-\frac{t}{\alpha_{0}}\right)
$$

and the mean time that the SU transmit with collision as shown in Fig. 2 may be expressed as

$$
\bar{y}(T)=\int_{0}^{T-\tau}(T-\tau-t) P(t) d t=T-\tau-\alpha_{0}\left(1-\exp \left(-\frac{T-\tau}{\alpha_{0}}\right)\right)
$$

where the mean time that the SU transmit with collision is a function of frame length $T$. Finally, we can calculate $P_{p}^{s}[16]$ as

$$
P_{p}^{s}=\frac{\bar{y}(T)}{T-\tau}=1-\frac{\alpha_{0}}{T-\tau}\left(1-\exp \left(-\frac{T-\tau}{\alpha_{0}}\right)\right)
$$

By substituting (4) and (10) into (6), the normalized throughput of the SU with $K$ PUs coexisting in the network can now be expressed as

$$
R_{p}(K, T)=\left(1-\beta^{K}\right) \frac{\alpha_{0}}{T}\left(1-\exp \left(-\frac{T-\tau}{\alpha_{0}}\right)\right)
$$

\section{B. Throughput of $S U$ with Imperfect Spectrum Sensing}

In real time environment, spectrum sensing is always imperfect and the sensing errors may be categorized as (i) errors due to missed detection and (ii) errors due to false alarm. Missed 
detection leads to more collisions between the SU and the PUs where the channel is wrongly considered idle while the false alarm makes the SU keep silent even if the idle channel is available to SU. Let $p_{m d}$ and $p_{f a}$ denote the probability of missed detection and the probability of false alarm respectively for each sensing result to each channel. Here, if missed detection occurs, there is a probability that the PU becomes idle (inactive) before the current frame ends, which gives very limited contribution to the throughput of the SU. Fig. 4 is a graphical illustration to show this limited contribution to the normalized throughput due to missed detection.

In order to obtain the expression of the accurate normalized throughput of the SU, firstly it is necessary to calculate the probability of collision due to missed detection $P_{i p}^{s}$. We define $r$ as the time required for PU to become idle from the starting point of data transmission slot in the current frame as also shown in Fig. 4. The collision free duration of the current frame with PU can thus be expressed as

$$
z(r)= \begin{cases}T-\tau-r, & 0 \leq r \leq(T-\tau) \\ 0, & r \geq(T-\tau)\end{cases}
$$

Because the random variable $r$ is also exponentially distributed, the PDF of $r$ is

$$
Q(r)=\frac{1}{\alpha_{1}} \exp \left(-\frac{r}{\alpha_{1}}\right)
$$

and with missed detection the mean time that the SU transmits without collision as shown in Fig. 4 may be expressed as

$$
\bar{z}(T)=\int_{0}^{T-\tau}(T-\tau-r) Q(r) d r=T-\tau-\alpha_{1}\left(1-\exp \left(-\frac{T-\tau}{\alpha_{1}}\right)\right) .
$$

The percentage of collision transmission out of data transmission duration is

$$
P_{i p}^{s}=\frac{T-\tau-\bar{z}(T)}{T-\tau}=\frac{\alpha_{1}}{T-\tau}\left(1-\exp \left(-\frac{T-\tau}{\alpha_{1}}\right)\right)
$$

Then we may start to conclude the expression of normalized throughput of SU with imperfect spectrum sensing. Similarly, for any sensing instant, we still assume that $i$ indicates the number of occupied channels while $K-i$ indicates the number of free channels, where $i=0, \ldots, K$. 
After sensing, we denote $m$ as the number of missed detected channels out of $i$ occupied channels and $n$ as the number of false alarmed channels out of $K-i$ free channels. In this case, $K-i-n$ indicates the number of correctly sensed free channel while $i-m$ indicates the number of correctly sensed occupied channel. For any given $i$, the probability that there are $n$ sensed channels with false alarm out of $K-i$ actual free channels and the probability that there are $m$ sensed channels with missed detection out of $i$ actual occupied channels are given by

$$
\begin{gathered}
P_{(n \mid K, i)}=\left(1-p_{f a}\right)^{K-i-n} p_{f a}^{n}, \\
P_{(m \mid K, i)}=\left(1-p_{m d}\right)^{i-m} p_{m d}^{m},
\end{gathered}
$$

respectively, where $m=0, \ldots, i$ and $n=0, \ldots, K-i$. Consequently, for any sensing instant, the joint probability that there are $i$ channels are actually occupied ( $K-i$ channels are actually free) and after sensing there are $n$ sensed channels with false alarm out of $K-i$ actual free channels at same time there are $m$ sensed channels with missed detection out of $i$ actual occupied channels could be expressed using chain rule of conditional probability [22] as:

$$
P_{(K, i, m, n)}=P_{(K, i)} P_{(n \mid K, i)} P_{(m \mid K, i)},
$$

where $P_{(K, i)}$ is given as (3). If $K-i-n=0$ and at the same time $m=0$, which means all busy channels are sensed correctly and all free channels have false alarm, the SU will keep silent and there is no throughput contribution, otherwise the SU will transmit data during data transmission slot using one of the $K-i-n$ correctly sensed free channels or $m$ missed detected busy channel as the SU is supposed to choose the best channel to transmit. It is assumed all PUs and the SU are randomly located in a unit area and also channel quality is varying with time and location. Therefore, we can say the best channel would probably be any one of all channels, which means choosing the best channel is equivalent to randomly choosing a channel out of all sensed free channels if we consider huge amount of Monte Carlo Simulations. Thus, the probability that 
one of missed detected busy channels would be chosen by the SU is $\frac{m}{K-i-n+m}$ and similarly the probability that one of correctly sensed free channels would be chosen is $\frac{K-i-n}{K-i-n+m}$. For any given $i, m$ and $n$, we calculate the normalized throughput of the SU as:

$R_{i p}^{i, m, n}(K, T)=P_{(K, i, m, n)} L$

where

$$
L= \begin{cases}0, & \text { if } K-i-n+m=0 \\ \frac{T-\tau}{T}\left(\left(\frac{K-i-n}{K-i-n+m}\right)\left(1-P_{p}^{s}\right)+\frac{m}{K-i-n+m}\left(1-P_{i p}^{s}\right)\right), & \text { if } K-i-n+m \neq 0\end{cases}
$$

In order to calculate the overall normalized throughput, all combinations of $i, m, n$ should be considered. Then using the law of total probability [22], the normalized throughput of the SU with imperfect spectrum sensing can be final expressed as:

$$
\begin{aligned}
R_{i p}(K, T) & =\sum_{i=0}^{K} C_{K}^{i} \sum_{n=0}^{K-i} \sum_{m=0}^{i} C_{K-i}^{n} C_{i}^{m} R_{i p}^{i, m, n}(K, T) \\
& =\sum_{i=0}^{K} C_{K}^{i} P_{(K, i)} \sum_{n=0}^{K-i} \sum_{m=0}^{i} C_{K-i}^{n} C_{i}^{m} P_{(n \mid K, i)} P_{(m \mid K, i)} L
\end{aligned}
$$

by substituting(3), (16) and (17) into (21), then

$$
=\sum_{i=0}^{K} C_{K}^{i}(1-\beta)^{K-i} \beta^{i} \sum_{n=0}^{K-i} \sum_{m=0}^{i} C_{K-i}^{n} C_{i}^{m} p_{f a}^{n}\left(1-p_{f a}\right)^{K-i-n} p_{m d}^{m}\left(1-p_{m d}\right)^{i-m} L,
$$


where $C_{b}^{a}=\frac{b !}{(b-a) ! a !}$ and by substituting(10) and (15) into (20), then $L$ could be expressed as

$$
L=\left\{\begin{array}{rr}
0, & \text { if } K-i-n+m=0 \\
\frac{\alpha_{0}(K-i-n)}{T(K-i-n+m)}\left(1-\exp \left(-\frac{T-\tau}{\alpha_{0}}\right)\right)+\frac{m(T-\tau)}{T(K-i-n+m)}-\frac{m \alpha_{1}}{T(K-i-n+m)}\left(1-\exp \left(-\frac{T-\tau}{\alpha_{1}}\right)\right), \\
\text { if } K-i-n+m \neq 0
\end{array}\right.
$$

\section{Simulation Results and Discussions}

We simulate the cognitive radio network under perfect and imperfect spectrum sensing environments with multiple PUs to determine the impact of imperfect sensing, variable frame length and variable PUs' traffic load on normalized throughput. 20000 Monte Carlo simulations are performed and the simulation settings of cognitive radio transmission are described as follows:

- $\mathrm{PU}$ transmission rate $=20 \mathrm{~ms} /$ packet.

- $\mathrm{SU}$ transmission rate $=1 \mathrm{~ms} /$ packet.

- The sensing length $\tau=1 \mathrm{~ms}$.

- Two types of traffic loads are considered:

1) Normal traffic load, i.e. Voice over Internet Protocol (VOIP) traffic with $\alpha_{0}=650 \mathrm{~ms}$, $\alpha_{1}=352 \mathrm{~ms}$ and $\beta=0.35$, i.e. $35 \%$ traffic load (this is considered as a benchmark PU traffic load in the following discussions).

2) Heavy traffic load with $\alpha_{0}=\alpha_{1}$ and $\beta=0.5$, i.e. $50 \%$ traffic load.

\section{A. Effect of Variable Number of PUs on Throughput}

In this section, we investigate the impact of multiple PUs in cognitive radio networks under prefect and imperfect spectrum sensing and variable PU traffic loads. Simulation results are discussed and compared with analytical results later in this section.

Fig. 5(a) shows the probability of collision for SU under the normal and the heavy traffic environments with perfect spectrum sensing. The probability is calculated as the ratio of number 
of collided packets to the total number of transmitted packets during the cognitive radio transmission. The probability of packet collisions is found higher under heavy traffic load since more frequent packets transmissions lead to more collisions. It should be noted that under the perfect spectrum sensing, the probabilities of collisions with variable number of PUs are considered as same since the cognitive radio network is under the assumption that the SU only use the best channel if more than one channels are sensed as idle.

Similarly, Fig. 5(b) shows the probability of total collisions of SU with imperfect spectrum sensing and with multiple PUs coexisting in the network for VOIP traffic. It should be noted that the total collisions with imperfect spectrum includes two parts (i) the collisions when the PUs activate during the data transmission phase of the SUs, whose probability is calculated as (10) and (ii) the collisions due to missed detections, whose probability is calculated as (15). Here, we assume that the probability of missed detection $p_{m d}$ and probability of false alarm $p_{f a}$ are considered as fixed and both set to be $5 \%$. It can be seen that different from the perfect sensing case, various number of PUs result in different probabilities of collision. In comparison with Fig. 5(a), more collisions (approximately 4\%) occur due to the missed detections and the probability of these extra collisions is given by $P_{i p}^{s}$. However, the sensing errors caused by missed detection may be gradually tolerated with the increase in number of available channels. This can also be seen by comparing the two figures Fig. 5(a) and Fig. 5(b), i.e. the probability of total collisions with imperfect spectrum sensing approaches the $P_{p}^{s}$ with the increasing number of PU participating in the cognitive radio network. The same may be considered as a reasonable justification of why $P_{p}^{s}$ does not change with an increase in the number of coexisting PUs.

Fig. 6 shows the normalized throughput of SU as a function of various frame length for VOIP network traffic under the perfect and imperfect spectrum sensing environments. It can be observed clearly that the throughput of the SU increases with the increase in number of PUs which join the cognitive radio network. This is due to the fact that the SU has more opportunities to access channels in the cognitive radio network with multiple PUs coexisting. However, the throughput certainly suffers from imperfect sensing and the degradation is significant when perfect and imperfect sensing curves of each pair are compared. This degradation is not only due to more 
collisions but also the false alarm which makes the SU keep silent even if the channel is free. The degradation impact is slightly mitigated as number of PUs increases. For example, the imperfect sensing curve is closer with the perfect sensing curve for $K=5$ as compared with other pairs with comparatively less number of PUs. It also can be seen that an optimal frame length which gives the best throughput can be spotted which is approximately located at $T_{\text {opt }}=35 \mathrm{~ms}$.

Similarly, Fig. 7 illustrates the normalized throughput of the SU for heavy traffic $(\beta=50 \%)$ with perfect and imperfect spectrum sensing environments. Similar to the VOIP traffic load case, Fig. 7 illustrates the same trend of normalized throughput varying as the SU's frame length varies. Obviously, under heavy traffic load case the optimal frame duration moves to around $T_{\text {opt }}=28$ ms which offers the best normalized throughput of SU. The normalized throughput of the SU is worse than that of the VOIP traffic load scenario with any corresponding number of PUs because the channels are more often occupied by their licensed (legal users) PUs under heavy traffic load. This degradation can be clearly noticed by comparing the respective curves in Fig. 6 and Fig. 7. As an example, consider the curves with $K=2$ PUs participating in the network. Under perfect spectrum sensing, the normalized throughput of the SU with optimal frame length degrades from 0.83 offered by VOIP traffic load to 0.71 offered by heavy traffic load, i.e. $17 \%$ degradation due to increase in PU traffic load from $35 \%$ to 50\%. Similarly, under imperfect spectrum sensing the degradation due to the increase in PU traffic load increases to $19 \%$.

There is another non-intuitive result that can be deduced from comparison of Fig. 6 and Fig. 7. That is, for heavy traffic the impact of imperfect sensing on normalized throughput with variable number of PUs shows different trends compared with that for VOIP traffic. It can be seen that imperfect sensing curves become far from the corresponding perfect sensing curves with the increase in number of PUs in the network. This shows that how the throughput degradation due to imperfect sensing varies as the increase in number of PUs, mainly depends on the PUs' traffic loads. When PUs are under relatively high traffic load, imperfect sensing degrades more SU's throughput as PUs' number increasing while the degradation becomes less as PUs' number increases when PUs are under relatively low traffic load. We choose different traffic loads from $20 \%$ to $80 \%$ and summarize the trend of normalized throughput varying due to imperfect sensing 
as Table I. This result is very useful to determine how to set sensing parameters to achieve the required sensing accuracy because the throughput of the SU has shown the different sensitivity to sensing accuracy under different traffic loads of PUs.

Finally, it is also illustrated that the simulation results and the analytical results are in perfect agreement with both the perfect and imperfect spectrum sensing and under both types of traffic loads (compare the red markers with the respective solid and dashed black curves).

\section{B. Relative Throughput Gain at Optimal Frame Duration}

To further explore the effect of the variable number of PUs, we introduce a definition of relative throughput gain $(\zeta)$. For any given number of PU, $\zeta$ is defined as

$$
\zeta=\frac{\max \left\{R_{(\cdot)}\left(T_{o p t}\right)\right\}-\min \left\{R_{(\cdot)}(T)\right\}}{\min \left\{R_{(\cdot)}(T)\right\}},
$$

where $\max \left\{R_{(\cdot)}\left(T_{\text {opt }}\right)\right\}$ is the maximum normalized throughput achieved under perfect or imperfect spectrum sensing scenarios with SU's variable frame duration from $10 \mathrm{~ms}$ to $180 \mathrm{~ms}$, i.e. the achieved normalized throughput with optimal frame duration. Similarly, $\min \left\{R_{(\cdot)}(T)\right\}$ is the minimum normalized throughput achieved under perfect or imperfect sensing scenarios with this variable frame duration of the SU. The results reflected by both perfect and imperfect spectrum sensing under VOIP and heavy traffic loads are summarized in Table II for various number of PUs. It can been seen that the relative throughput gain with optimal frame duration is almost fixed as the number of PUs increases. Consistent with the result discussed in last subsection, the throughput gain at optimal frame length is mainly affected by traffic loads of PUs, i.e. under heavy traffic load it is always more pronounced than the gain under VOIP traffic load.

\section{Effect of Variable PU Traffic Load}

Based on previous discussions, one may reach to the conclusion that the increase in number of PUs does not affect the optimal frame duration while various PUs' traffic load does. Therefore, it would be more interesting to further explore the effect of variable PU activity on the optimal frame duration length. This may be considered as a case where the amount of traffic that PU 
generates is variable throughout the day or during a particular time period. For simplicity, we consider a case where the cognitive radio network comprises of a PU and a SU. Fig. 8(a) illustrates the effect of variable load factor on the optimal frame duration of SU which achieves the maximum normalized throughput. Here, we vary the load factor of PU $(\beta)$ from $20 \%$ to $90 \%$. It can be seen that the optimal frame duration of cognitive radio transmission decreases with the increase in PU load factor. This leads to a conclusion that in order to maximize the normalized throughput, the optimal frame length of the SU cognitive radio transmission should be adaptively changed as the variable traffic load of the PU. This is considered as a useful investigation which is equivalently important for standardization of cognitive radio networks specially for a case where the PU traffic load (PU activity) is variable.

Now, we investigate the impact of variable PU traffic load on the interference to the PU due to SU cognitive radio transmission. The interference is due to the packet collision and the corresponding probability of collision is shown in Fig. 8(b) as a function of variable PU traffic load with their corresponding optimal frame lengths shown in Fig. 8(a). It can be seen clearly that the interference levels to the PUs decreases with the increase in PU traffic load. The reason is that with the increasing PU traffic load although collisions due to missed detection increase, the amount of collisions when the PUs activate during the data transmission phase of the SU becomes much less, which dominates the total amount of collisions and thus the interference level decreases. To control the interference level of PU to a tolerable level, an appropriate frame duration should be selected subject to different system requirement and achievable throughput. In other words, a tradeoff problem between achievable throughput of the SU and the interference to PUs needs to be considered when the frame duration length of the SU is set for a cognitive radio network.

\section{Conclusions}

In this paper, we analyzed the throughput of the SU in a cognitive radio network based on the frame structure which consists of sensing and data transmission slots. The achievable normalized throughput of the SU has been analytically expressed with multiple PUs under perfect and 
imperfect spectrum sensing environments. The impact of coexistence of multiple PUs in the network and subsequently variable frame length, on the achievable normalized throughput has been critically illustrated for perfect and imperfect spectrum sensing scenarios with variable traffic loads of PUs. The analysis with imperfect spectrum sensing is very useful to determine the required sensing accuracy during the cognitive radio transmission of SU. The behavior of the imperfect sensing curve has been investigated and it has been found that the impact of imperfect sensing on throughput is not only related to the number of coexisting PUs but also much involved with the traffic loads of a realistic cognitive radio network. It has been illustrated that the analytical and the simulation results are in perfect agreement under all considered scenarios. Moreover, it has also been shown that the optimal frame length which maximizes the throughput of the SU strictly depends on the PU traffic load. However, the proper frame length should be adaptively chosen to achieve higher throughput of the SU at the same time to control the interference to the legal PUs at a tolerable level.

\section{REFERENCES}

[1] Federal Communication Commission: 'Spectrum policy task force report, FCC 02-155, November 2002

[2] Mitola III J., Maguire Jr., G. Q.: 'Cognitive radio: making software radios more personal', IEEE Jour. Personal Communications, Augest 1999, 6, (4), pp. 13-18

[3] Haykin S.: 'Cognitive radio: brain-empowered wireless communications', IEEE Jour. Selected Areas in Communications, February 2005, 23, (2), pp. 201-220

[4] Zhao Q., Sadler B. M.: 'A Survey of Dynamic Spectrum Access', IEEE Mag. Signal Processing, May 2007, 24, (3), pp. 79-89

[5] Akyildiz I. F., Lee W.-Y., Vuran, M. C., Mohanty, S.: 'A survey on spectrum management in cognitive radio networks', IEEE Mag. Communications, April 2008, 46, (4), pp. 40-48

[6] Digham F. F., Alouini M.-S., Simon M. K.: 'On the energy detection of unknown signals over fading channels', IEEE Trans. Communications, January 2007, 55, (1), pp. 21-24 
[7] Zeng Y., Liang Y.-C.: 'Eigenvalue-based spectrum sensing algorithms for cognitive radio', IEEE Trans. Communications, June 2009, 57, (6), pp. 1784-1793

[8] Penna F., Garello, R., Figlioli, D., Spirito, M. A.: 'Exact non-asymptotic threshold for eigenvalue-based spectrum sensing', Cognitive Radio Oriented Wireless Networks and Communications, 2009. CROWNCOM '09. 4th International Conference on, June 2009, pp. $1-5$

[9] Yucek T., Arslan H.: 'A survey of spectrum sensing algorithms for cognitive radio applications', IEEE Communications Surveys and Tutorials, 2009, 11, (1), pp. 116-130

[10] Shakir M. Z., Rao A., Tang W., Imran M. A., Alouini M.-S.: 'Eigenvalue ratio detection based on exact analytical moments of smallest and largest eigenvalues', Proc. ICST Conf. Cognitive Radio Oriented Wireless Networks and Communications, CrownCom'2011, Osaka, Japan, May 2011

[11] Shakir M. Z., Rao A., Alouini M.-S.: 'Collaborative spectrum sensing based on the ratio between largest eigenvalue and Geometric mean of eigenvalues', Proc. Intl. Conf. Global Communications., GLOBECOM'2011, Houston, TX USA December 2011

[12] Liang Y. C., Zeng Y., Peh E. C. Y., Hoang, A. T.: 'Sensing-Throughput Tradeoff for Cognitive Radio Networks', 2007 IEEE International Conference on Communications, June 2007, pp. 5330-5335

[13] Liang Y. C., Zeng Y., Peh E. C. Y., Hoang, A. T.: 'Sensing-Throughput Tradeoff for Cognitive Radio Networks', IEEE Trans. Wireless Communications, April 2008, 7, (4), pp. $1326-1337$

[14] Pei Y., Liang Y.-C., Teh K. C., Li K. H.: 'Sensing-throughput tradeoff for cognitive radio networks: A multiple-channel scenario', IEEE 20th International Symposium on Personal, Indoor and Mobile Radio Communications, September 2009, pp. 1257-1261

[15] Stotas S., Nallanathan A.: 'Optimal Sensing Time and Power Allocation in Multiband Cognitive Radio Networks', IEEE Transactions on Communications, January 2011, 59, (1), pp. 226-235

[16] Pei Y., Hoang A. T., Liang Y.-C.: 'Sensing-Throughput Tradeoff in Cognitive Radio 
Networks: How Frequently Should Spectrum Sensing be Carried Out?', IEEE 18th Intl. Symp. on Personal, Indoor and Mobile Radio Communications, PIMRC'2007, September 2007, pp. $1-5$

[17] Goldsmith A.: 'Wireless Communications', Cambridge University Press, New York, 2003

[18] IEEE 802.22: 'Working Group on Wireless Regional Area Networks', Available at http://ieee802.org/22/, 2011

[19] Zhao Q., Tong L., Swami A., Chen Y.: 'Decentralized cognitive MAC for opportunistic spectrum access in ad hoc networks: A POMDP framework', IEEE Journal on Selected Areas in Communications, April 2007, 25, (3), pp. 589-600

[20] Jiang S., Chao L.-H., Chao H.-L.: 'A decentralized MAC protocol for cognitive radio networks', 2011 IEEE Conference on Computer Communications Workshops (INFOCOM WKSHPS), April 2011, pp. 24-29

[21] Issariyakul T., Pillutla L. S., Krishnamurthy V.: 'Tuning radio resource in an overlay cognitive radio network for TCP:greed Isn't good', IEEE Mag. Communications, July 2009, 11, (1), pp. 57-63

[22] Papoulis A. and Pillai S. U.: 'Probability, Random Variables and Stochastic Processes', McGraw-Hill, New York, 2002 


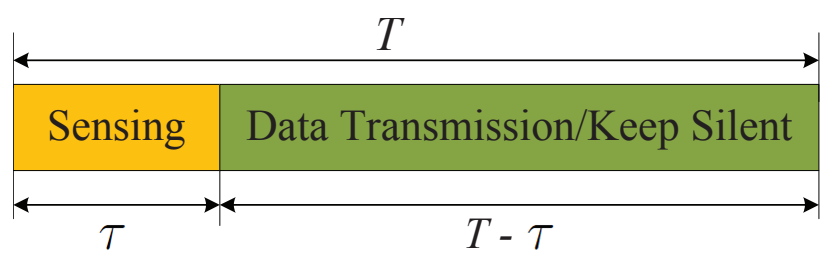

Fig. 1. A graphical structure of a typical frame structure of SU cognitive radio transmission.

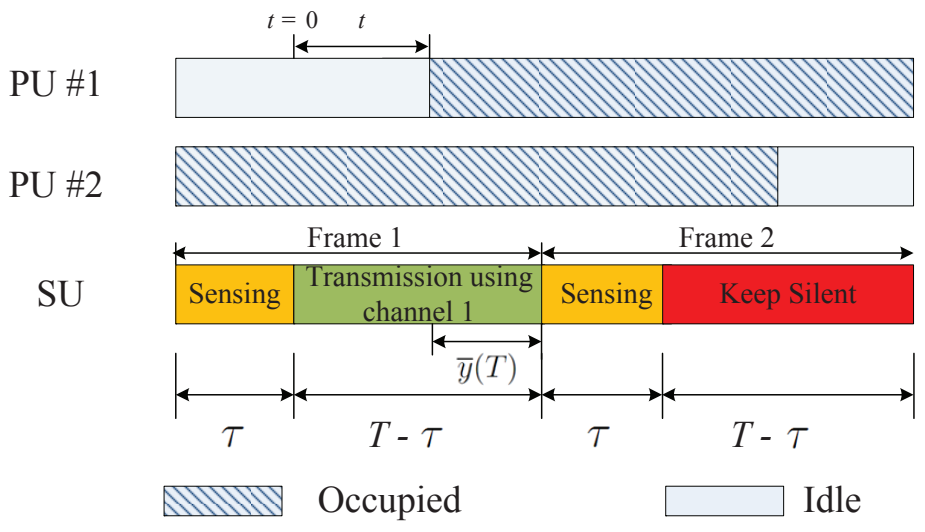

Fig. 2. A graphical structure of a typical cognitive radio transmission with $K=2$ PUs with perfect sensing.

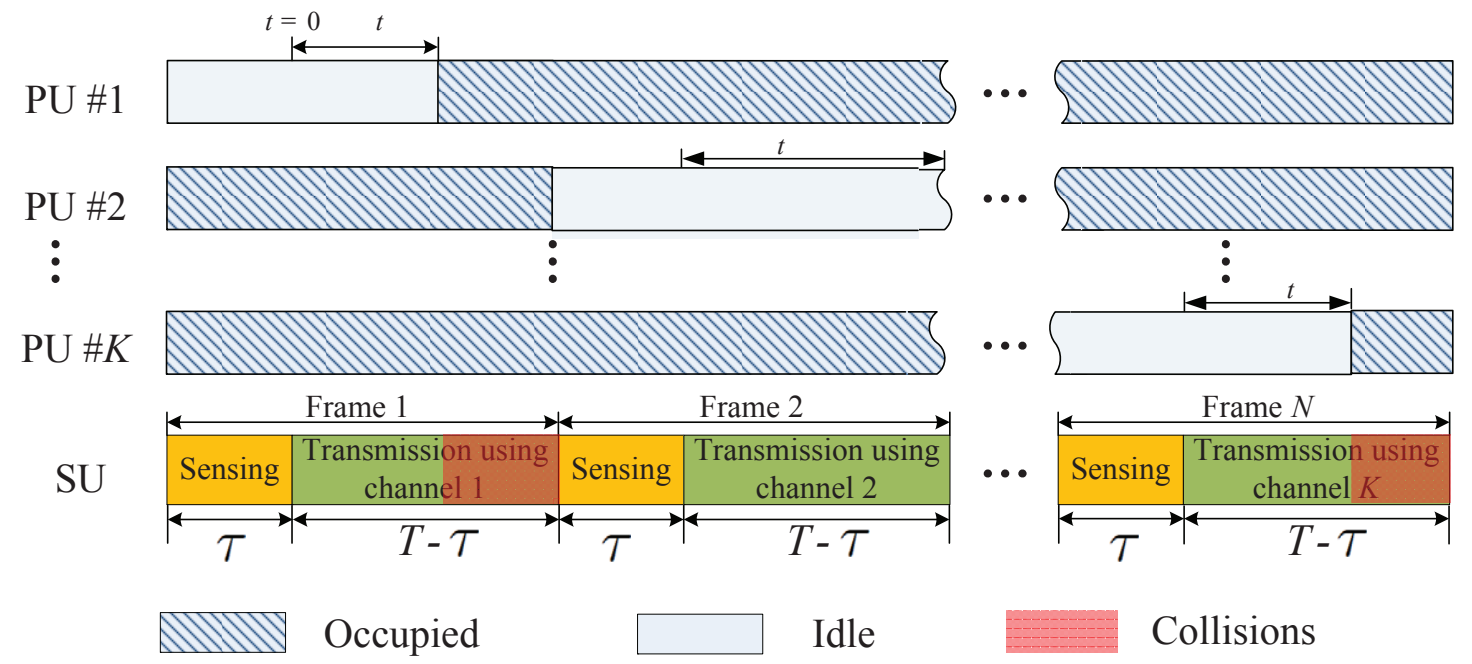

Fig. 3. A graphical illustration of data packets collision when PU becomes active in a typical cognitive radio transmission with perfect sensing. 


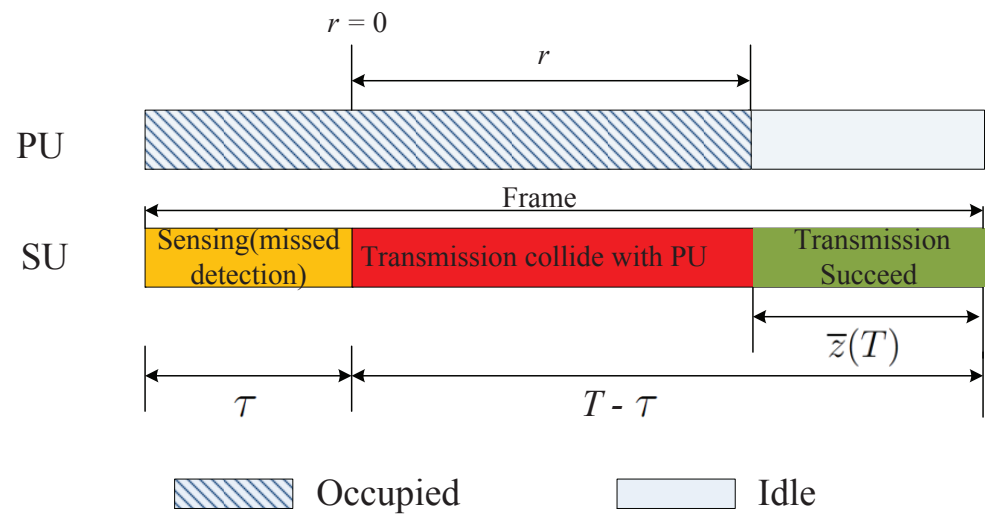

Fig. 4. A graphical illustration of a typical cognitive radio transmission with missed detection. 


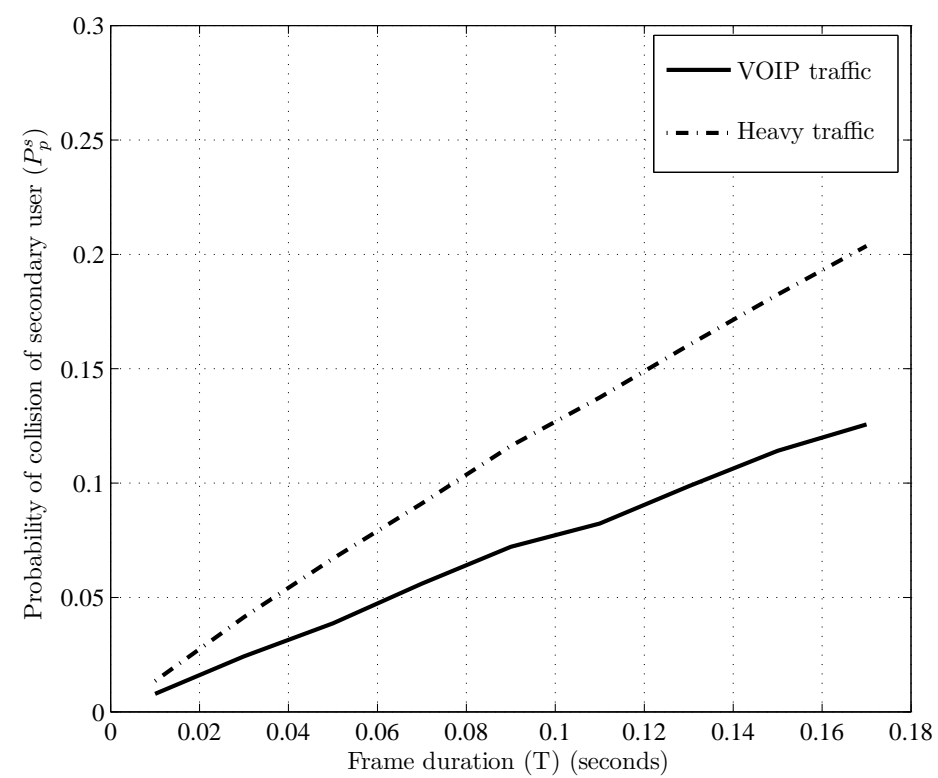

(a)

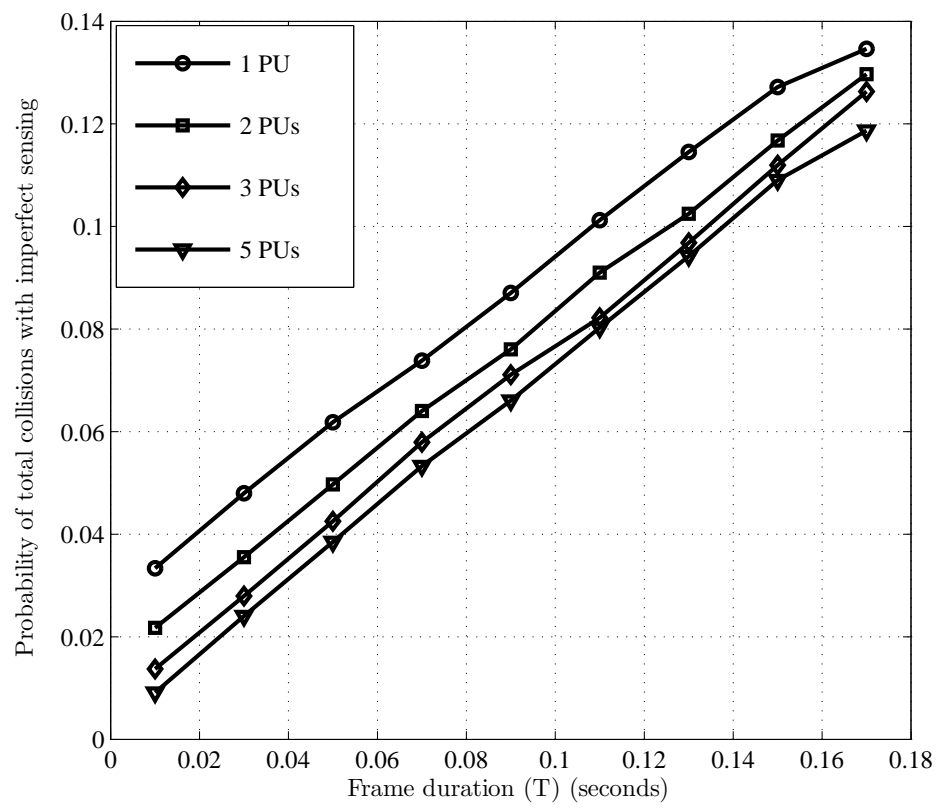

(b)

Fig. 5. Probability of total collision of SU for: (a) perfect spectrum sensing under VOIP and heavy traffic; and (b) imperfect spectrum sensing under VOIP traffic. 


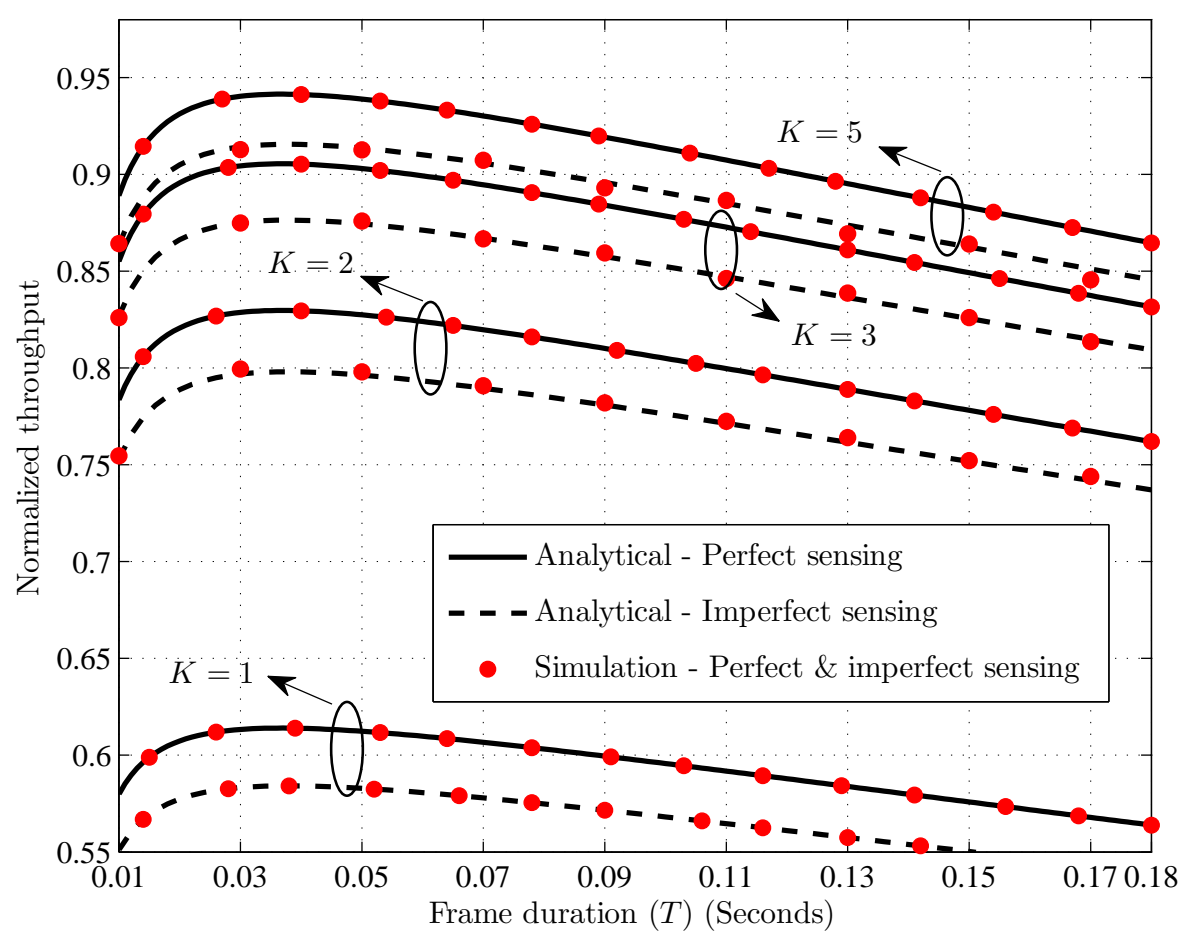

Fig. 6. Normalized throughput vs. frame duration for VOIP traffic showing; (i) impact of perfect and imperfect spectrum sensing on normalized throughput (compare solid curves with dashed curves for selected values of primary users $K$ ) and (ii) comparison between simulation and analytical results (compare solid and dashed curves with the respective red dotted markers).

TABLE I

SU'S THROUGHPUT DEGRADATION DUE TO IMPERFECT SENSING

\begin{tabular}{|c|c|c|c|c|c|c|c|}
\hline \multirow{2}{*}{$\beta$} & $20 \%$ & $30 \%$ & $40 \%$ & $50 \%$ & $60 \%$ & $70 \%$ & $80 \%$ \\
\hline 1 & $3.71 \%$ & $3.23 \%$ & $2.75 \%$ & $2.27 \%$ & $1.80 \%$ & $1.32 \%$ & $0.86 \%$ \\
\hline 2 & $2.28 \%$ & $2.94 \%$ & $3.31 \%$ & $3.41 \%$ & $3.23 \%$ & $2.78 \%$ & $2.06 \%$ \\
\hline 3 & $1.50 \%$ & $2.45 \%$ & $3.32 \%$ & $3.98 \%$ & $4.30 \%$ & $4.15 \%$ & $3.43 \%$ \\
\hline 5 & $1.15 \%$ & $2.05 \%$ & $3.15 \%$ & $4.41 \%$ & $5.59 \%$ & $6.37 \%$ & $6.18 \%$ \\
\hline
\end{tabular}




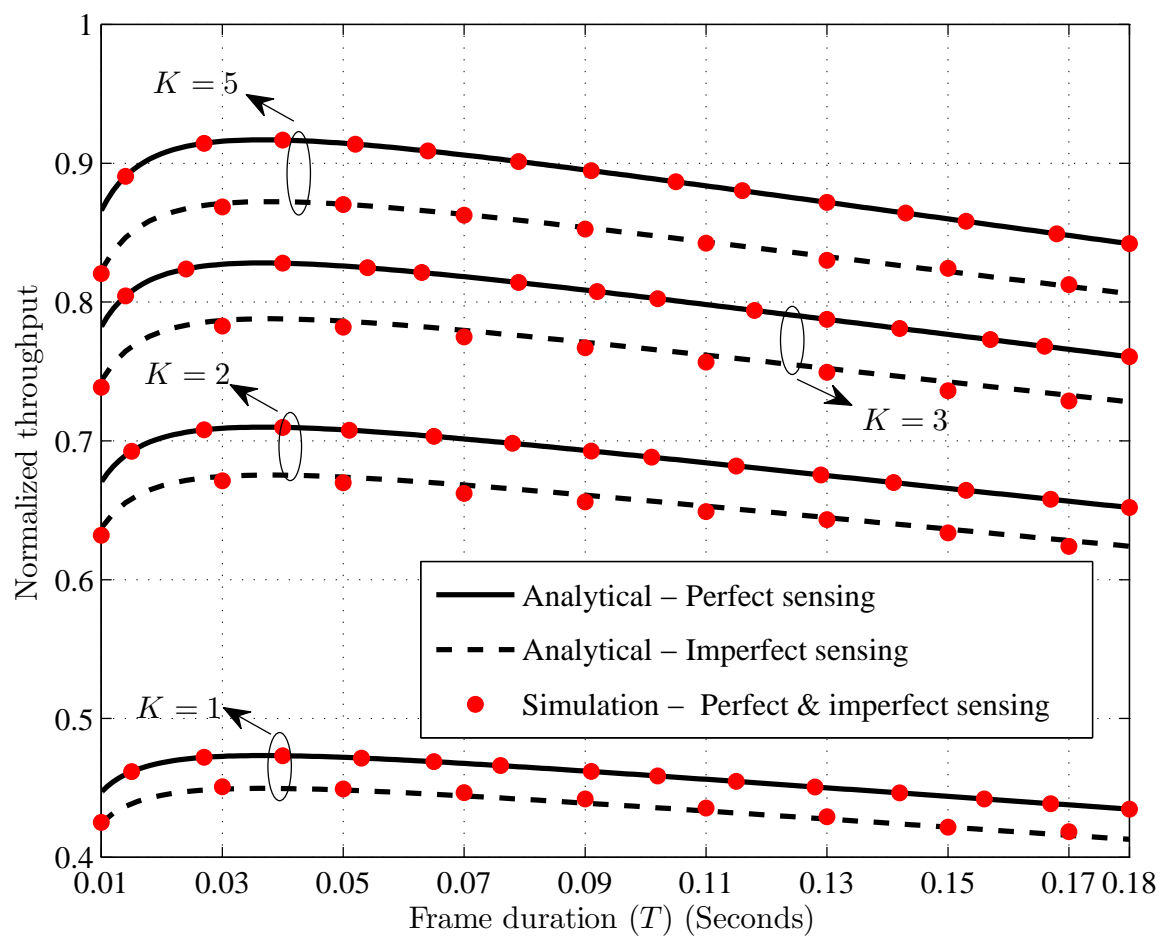

Fig. 7. Normalized throughput vs. frame duration for heavy traffic (i.e. 50\% PU traffic load )showing; (i) impact of perfect and imperfect spectrum sensing on normalized throughput (compare solid curves with dashed curves for selected values of primary users $K$ ) and (ii) comparison between simulation and analytical results (compare solid and dashed curves with the respective red dotted markers).

TABLE II

RELATIVE Throughrut GAIN WITH OPTIMAL FRAME LENGTH

\begin{tabular}{|c|c|c|c|c|c|}
\hline \multicolumn{3}{|c|}{ VOIP traffic } & \multicolumn{3}{c|}{ Heavy traffic } \\
\hline Number of PUs & Perfect Sensing & Imperfect Sensing & Number of PUs & Perfect Sensing & Imperfect Sensing \\
\hline 1 & $6.92 \%$ & $7.58 \%$ & 1 & $9.21 \%$ & $8.02 \%$ \\
\hline 2 & $7.75 \%$ & $8.00 \%$ & 2 & $8.59 \%$ & $8.69 \%$ \\
\hline 3 & $7.51 \%$ & $7.55 \%$ & 3 & $8.40 \%$ & $8.21 \%$ \\
\hline 5 & $8.19 \%$ & $8.44 \%$ & 5 & $8.81 \%$ & $8.93 \%$ \\
\hline 10 & $7.22 \%$ & $7.31 \%$ & 10 & $8.92 \%$ & $8.73 \%$ \\
\hline
\end{tabular}




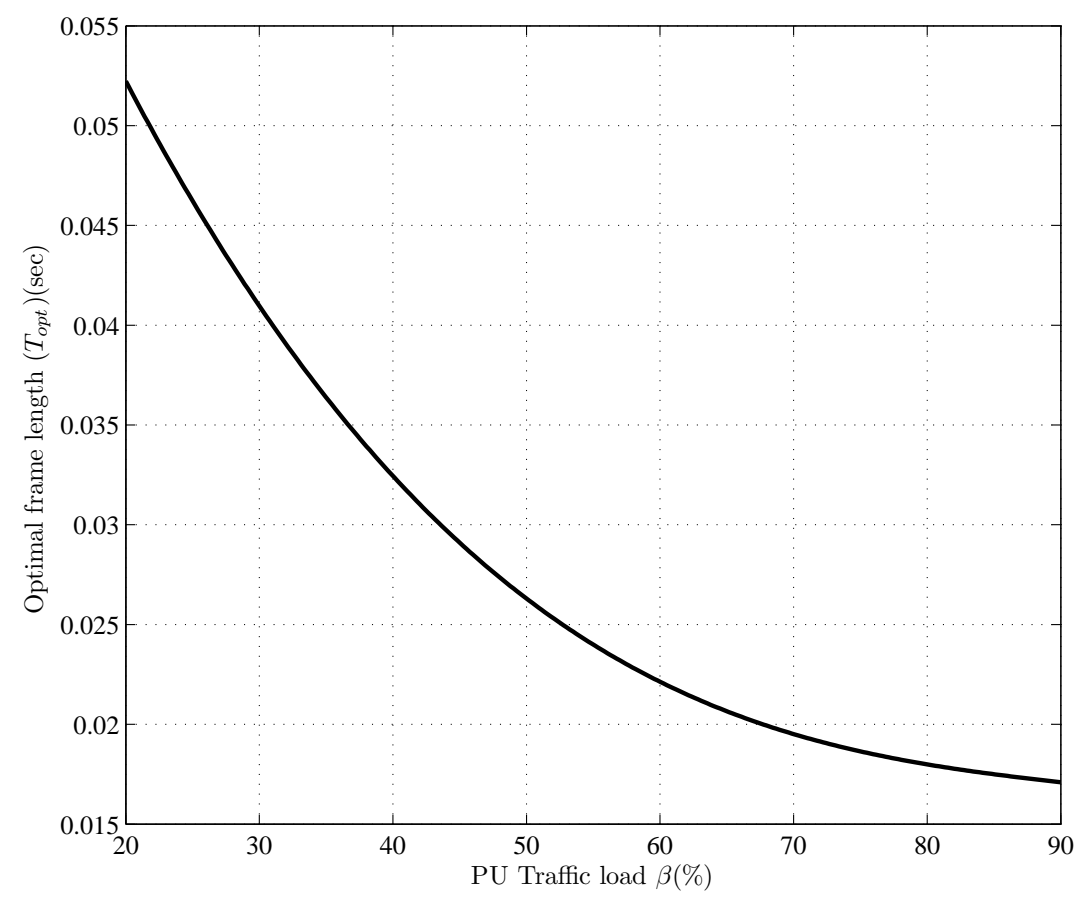

(a)

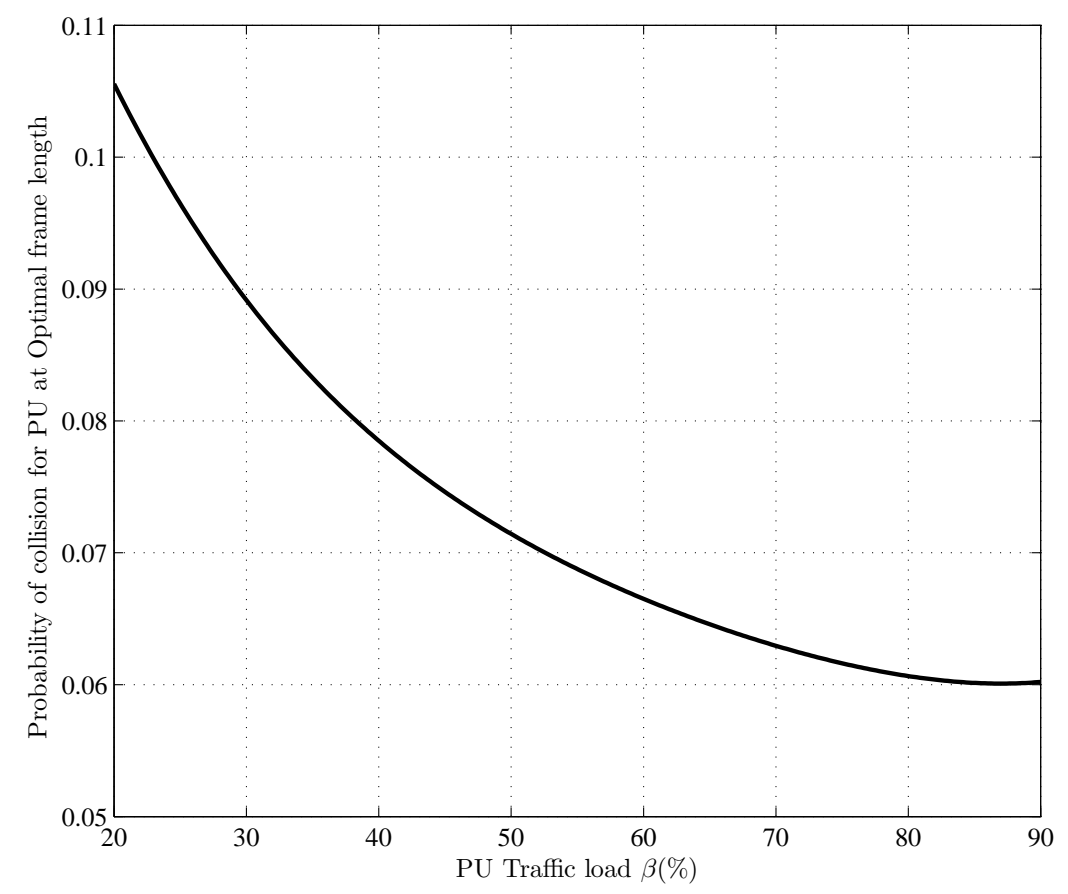

(b)

Fig. 8. Impact of variable traffic load on: (a) optimal frame duration; and (b) interference levels to the PUs caused by SU (probability of collision). 